Augmented Reality, Planung, Bürgerinformation

Im fünften Block kamen zwei interessante Randthemen zur Sprache: Prof. Dr. Jörg Blankenbach von der RWTH Aachen gab einen Überblick über Möglichkeiten zur Indoor-Positionierung und veranschaulichte seine Algorithmen. Frau Prof. Dr. Ingrid Jaquemotte von der Jade Hochschule Oldenburg schließlich gab der Vielfalt an Headworn Displays in Verbindung mit Virtual und Augmented Reality eine Struktur.

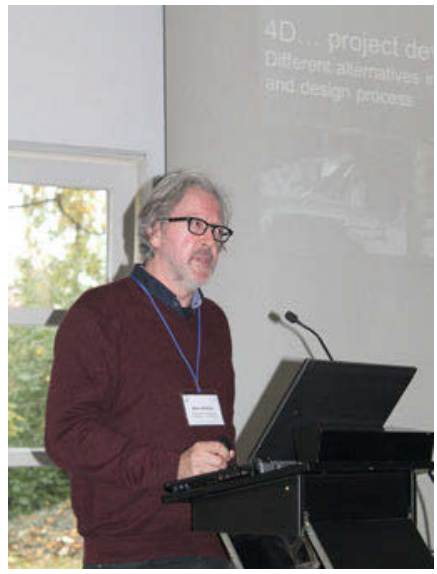

Mario Matthys, City of Ghent Belgien

Den Schluss der Veranstaltung bildete, wie in den vergangenen Jahren, der Blick über die bundesdeutsche Grenze ins benachbarte Ausland. Ing. Pascal Peter, Zürich, und Dipl.-Geoinf. Christian Dahmen, con terra $\mathrm{GmbH}$, Münster, schilderten die Anwendungen des Züricher Stadtmodells für Lärmberechnungen, Solarpotenziale, Mobilfunk und Flachdachbegrünung. 3D-Druck in Verbindung mit einer AR-App kam ebenso zur Sprache wie, ausführlicher, die Visualisierung im Web mit Hilfe von Streamingtechniken. Ein Höhepunkt der Veranstaltung war sicherlich der Vortrag von Mario Matthys, City of Ghent, Belgien, der die vielfältigen Möglichkeiten zur Nutzung von 3D-Modellen in einer ganzheitlichen Betrachtung im Rahmen der Stadtplanung und Bürgerinformation aufzeigte. Frau Prof. Jantien Stoter von der Uni Delft, Niederlande, gab zum Abschluss einen Überblick über die Modellierungserfolge einiger

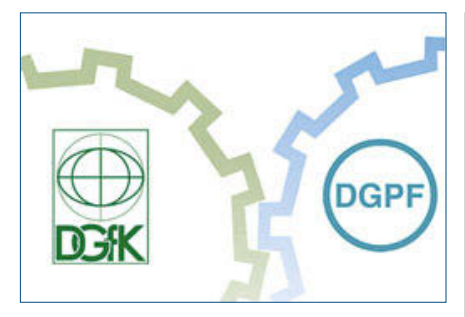

in der EuroSDR vertretenen Länder.

Die Vorträge können unter http:// www.3d-stadtmodelle.org/index. php?do=rue\&do2 =ws $14 /$ heruntergeladen werden. Mit erneuten 80 Teilnehmern waren die fröhlichen Kommissionsleiter auch dieses Jahr wieder sehr zufrieden. Wir hoffen, dass es den Teilnehmern ebenso ging.

Bettina Petzold, Wiesbaden Ekkehard Matthias, Hamburg

\section{Deutscher Kongress für Geographie 2015}

\section{Ankündigung und Call for Papers}

Anfang Oktober 2015 findet in Berlin der von der Deutschen Gesellschaft für Geographie (DGfG) veranstaltete Deutsche Kongress für Geographie (DKG 2015) statt. Insbesondere in den Fachsitzungen zur Didaktik der Geographie, deren Leitthemen über die Konferenz-Homepage einsehbar sind, wird sehr häufig die Kartographie thematisiert. Dies beweist, dass die Verwendung von Karten im Unterricht, egal ob elektronisch oder analog, von eminenter Bedeutung für die geographische Bildung ist. Die Vielzahl der Sitzungen mit kartographischem Bezug zeigt, wie wichtig unser Fach für die Nachbardisziplin Geographie ist.

Gegenwärtig besteht bis zum 11 . Januar 2015 die Möglichkeit, Beiträge
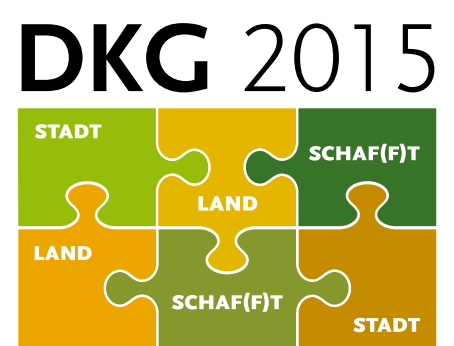

für die Fachsitzungen einzureichen. Für die Kartographie von besonderem Interesse ist die Sitzung DI-FS-12 "Von der analogen zur digitalen Karte - Der fundamentale Wandel der Kartographie und seine Bedeutung für geographisches Lehren und Lernen". Diese Session wird von Prof. Mark Vetter und Gymnasiallehrer Dr. Friedrich Barnikel gemeinsam geleitet. Vor dem Hintergrund der gegenwärtig stattfindenden grundlegenden Veränderungen in Kartographie und Kartographiedidaktik bietet sie ein Forum, um etwaige Implikationen für den Unterricht und auch für die Weiterentwicklung der Kartographie zu diskutieren. Die Veranstalter würden sich freuen, wenn das Thema bei den Leserinnen und Lesern der KN auf Interesse stößt und laden sie ein, hierzu Beiträge einzureichen. Näheres siehe unter www.dkg2015. hu-berlin.de

Mark Vetter, Karlsruhe

Friedrich Barnikel, München

\section{Hochschul-}

\section{nachrichten}

\section{Promotion \\ Stefan Hahmann}

\section{Am 12.6.2014 promovierte}

Herr Dipl.-Ing. (FH) Stefan Hahmann an der Fakultät Umweltwissenschaften, Institut für Kartographie, der Technischen Universität Dresden zum Thema „Zur Beziehung von Raum und Inhalt nutzergenerierter geographischer Informationen" zum Dr.-Ing. Die Gutachter waren Prof. Dr. Dirk Burghardt, TU Dresden (1. Gutachter), Prof. (em.) Dr. Bernd Teichert, Dresden (2. Gutachter), Prof. Dr. Ross Purves, Universität Zürich (3. Gutachter).

\section{Autorenreferat}

Während der vergangenen zehn Jahre vollzog sich eine wesentliche
Veränderung des World Wide Webs, das sich zum sogenannten "Web 2.0" entwickelte. Das wesentlichste Merkmal dieser neuen Qualität des WWW ist die Beteiligung der Nutzer bei der Erstellung der Inhalte. Diese Entwicklung fördert das Entstehen von Nutzergemeinschaften, die kollaborativ in unterschiedlichsten Projekten Informationen sammeln und veröffentlichen. Prominente Beispie-le für solche Projekte sind die Online-Enzyklopädie "Wikipedia", die Microblogging-Plattform "Twitter", die Foto-Plattform "Flickr" und die Sammlung topographischer Informationen "OpenStreetMap". Nutzergenerierte Inhalte, die direkt oder indirekt raumbezogen sind, können spezifischer als „nutzergenerierte geographische Informationen" bezeichnet werden. Der Raumbezug dieser Informationen entsteht entweder direkt durch die Angabe räumlicher Koordinaten als Metainformationen oder er kann indirekt durch die Georeferenzierung von in den Informationen enthaltenen Toponymen oder Adressen hergestellt werden.

Nutzergenerierte geographische Informationen haben für die Forschung den besonderen Vorteil, dass sie einerseits häufig gänzlich ohne oder nur mit geringen Kosten verfügbar gemacht werden können und andererseits eine Vielzahl von menschlichen Entscheidungen widerspiegeln, die mit dem Raum verknüpft sind. In der vorliegenden Dissertation wird die Beziehung von Raum und Inhalt nutzergenerierter geographischer Informationen aus zwei Perspektiven untersucht.

Im ersten Teil der Arbeit steht die Frage im Vordergrund, für welchen Anteil an Informationen eine Beziehung zwischen Raum und Informationsinhalt in der Art besteht, dass die Informationen im Georaum lokalisierbar sind. In diesem Zusammenhang existiert seit den 1980er Jahren die unter Nutzern von geographischen Informationssystemen weit verbreitete These, 